\title{
Dynamique interactionnelle au sein d'une formation privilégiant la participation et la création
}

\author{
Stéphane Allaire* et Christophe Reffay** \\ DOI https://doi.org/10.51657/ric.v3i1.41026
}

Résumé Dans un contexte de renouvellement de la pédagogie en enseignement supérieur, cet article présente d'abord les caractéristiques d'un programme de formation continue sur les classes multiâges en réseau privilégiant une métaphore de l'apprentissage qui s'inspire de la participation et de la création de connaissances. Ensuite, à partir d'échanges asynchrones qui se sont déroulés par l'entremise d'un forum électronique dans le cadre d'un des cours de ce programme portant sur l'intervention en classes multiâges en réseau, nous avons analysé la dynamique interactionnelle qui a pris forme entre les participants (7 étudiants et 1 formateur). Cette analyse a été menée à partir d'outils d'analyse semi-automatisée. Plus spécifiquement, un outil d'analyse du réseau social ainsi que la trousse Calico ont été utilisés. Les résultats mettent en lumière une implication importante des étudiants, bien qu'inégale entre eux, dans l'élaboration d'un discours collectif caractérisé par de nombreux ajouts d'idées qui ont aussi été mises en relief.

Mots-clés communauté d'apprenants, enseignement supérieur, analyse de réseaux sociaux, classe multiâge, télécollaboration, coélaboration de connaissances, outils d'analyse

\begin{abstract}
In a context where higher education's pedagogy is changing, this paper first presents characteristics of a training program about networked multi-grade classrooms that puts forward a learning metaphor based on participation and knowledge creation. Next, from asynchronous discourse elaborated with an electronic forum during one course's program about networked multi-grade classrooms, we analyzed interactions between participants (7 students and 1 trainer). This analysis was conducted using semi-automated analytic tools. Specifically, social network analysis tool and Calico applets were used. Results show an important implication from students, although unequal, at the elaboration of a collective discourse characterized by a lot of ideas addition that were also called in question.
\end{abstract}

Keywords community of learners, higher education, social network analysis, multi-grade classrooms, telecollaboration, knowledge building, learning analytics

\footnotetext{
* Université du Québec à Chicoutimi (UQAC)

** UMR STEF, École normale supérieure (ENS) de Cachan
} 


\section{Introduction et problématique}

Sfard (1998) identifie deux métaphores de l'apprentissage: une centrée sur l'acquisition et l'autre sur la participation. Historiquement, à l'exception des profils de type recherche menant à l'obtention d'un grade de maitrise ou de doctorat, l'université s'est passablement cantonnée derrière la première métaphore, qui conçoit cette institution éducative comme un lieu d'acquisition de connaissances, c'est-à-dire où des étudiants s'approprient l'état actuel du savoir humain; ou à tout le moins une partie de celui-ci. Des auteurs, tels Béchard (2001) et Brady et Eisler (1999), indiquent que de nos jours cette acquisition passe encore en majeure partie par des approches d'enseignement dites traditionnelles. On pense tout de suite à l'enseignement magistral, qui a la cote. Dans un tel modèle, le temps de parole appartient majoritairement à l'enseignant (Kozanitis \& Chouinard, 2009) qui, dit-on: «transmet des connaissances aux étudiants », et les interactions qui prennent forme au sein de la classe se traduisent par la séquence «question - réponse - rétroaction évaluative » (Cazden, 1988, 2001; Flanders, 1970; Sinclair \& Coulthard, 1975). Ainsi, l'enseignant adopte essentiellement une posture de transmission d'informations et, lorsqu'il y a des interactions verbales entre lui et les étudiants, c'est pour leur poser des questions visant à vérifier la compréhension; questions auxquelles un étudiant répond et dont la réponse est suivie d'une rétroaction de la part de l'enseignant afin d'en valider l'exactitude.

Des changements en cours depuis quelques décennies, notamment l'intensification de l'usage des TIC, suggèrent cependant une transformation du modèle pédagogique actuel de l'enseignement supérieur (Endrizzi, 2011) vers un modèle davantage axé sur une métaphore de la participation (Sfard, 1998), voire de la création (Pavoola, Lipponen \& Hakkarainen, 2004); à tout le moins pour les programmes de formation continue tels les programmes courts ou microprogrammes, les diplômes d'études supérieures spécialisées, etc. Dans cette lignée, Ramsden (2003) fait remarquer que la pression est de plus en plus forte en faveur d'une intégration des pédagogies dites actives en enseignement supérieur. Par métaphore de la participation, nous entendons un processus d'intégration et d'enculturation à une pratique culturelle établie. Quant à la métaphore de la création, elle fait référence à la combinaison d'expertises à des fins de résolutions de problèmes complexes. Il s'agit en quelque sorte d'une forme de créativité collaborative.

Nous soutenons qu'il existe au moins quatre raisons justifiant l'adoption de ces métaphores en enseignement supérieur, en complément à celle de l'acquisition qui, employée seule, apparaît de plus en plus désuète en regard du contexte et des connaissances qui prévalent aujourd'hui. Nous expliquons ci-après ces quatre motifs.

D'abord, sur le plan de l'accès à l'information, la démocratisation d'Internet et son ubiquité d'accès mettent en perspective la notoriété classique des institutions universitaires en tant que seuls véhicules des dernières découvertes scientifiques. Des universités semblent l'avoir compris depuis quelques années déjà. Pensons par exemple au Massachusetts Institute of Technology (MIT) et à son initiative OpenCourseWare qui donne accès gratuitement, en ligne, au contenu d'une gamme de cours qui y sont dispensés. Les perspectives sociocognitives de l'apprentissage (Laferrière \& Allaire, 2010) expliquent qu'une seule accessibilité à l'information et au contenu n'est pas garant d'un développement de connaissances et de compétences de qualité. Nous croyons qu'en offrant l'accès aux contenus, l'administration du MIT tente d'attirer les étudiants en montrant en quelque sorte toute l'importance des membres de sa communauté et la qualité des interactions dans cette communauté. C'est au contact de ses membres davantage qu'à celui d'informations que ces étudiants forgeront réellement leur esprit. C'est à tout le moins ce que laisse entendre l'énoncé suivant qui provient du site web de l'Institut: "MIT OpenCourseWare is a publication of the course materials that support the dynamic classroom interactions of an MIT education $»^{1}$.

Ensuite, sur le plan de la compréhension de la façon dont l'apprentissage humain s'effectue, la recherche en sciences cognitives appliquées, notamment les travaux du courant des

\footnotetext{
${ }^{1}$ http://ocw.mit.edu/about/
} 
sciences de l'apprentissage (learning sciences) (Bransford, Brown \& Cocking, 1999; Khine \& Saleh, 2010; Sawyer, 2005), met en exergue les caractéristiques d'un apprentissage en profondeur. Laferrière (2005) identifie diverses implications pour l'éducation des travaux issus de ce courant.

- Orienter l'apprentissage et le développement à partir de contextes promouvant la résolution de problèmes;

- Amener l'apprenant à mettre lui-même en action les procédures qui font l'objet d'un apprentissage;

- Ancrer les nouvelles connaissances à partir de celles que l'apprenant possède, ou croit posséder;

- Positionner les situations d'apprentissage au sein d'interactions sociales.

Cet argument, davantage épistémologique celui-là, contribue aussi à la remise en question du modèle pédagogique classique employé en enseignement universitaire.

Troisièmement, en contexte de formation continue des enseignants, la recherche sur le développement professionnel fournit une raison supplémentaire de questionner ce modèle. Alors que ce dernier envisage souvent l'apprentissage de contenus de façon encyclopédique et désincarnée de situations réelles et authentiques de mise en œuvre, nombre d'études ayant porté sur l'amélioration des pratiques enseignantes au cours des dernières décennies ont montré l'importance d'ancrer les nouveaux apprentissages à partir de situations actives et in situ (Butler, 2005 ; Rennie, 2001) et à faire en sorte que ces dernières soient cohérentes avec les pratiques qu'on souhaite voir émerger en classe (Garet, Porter, Desimone, Birman, \& Yoon, 2001). En outre, Darling-Hammond et Bransford (2005) soulignent l'importance d'axer une démarche de développement professionnel dans un contexte de communauté professionnelle.

Enfin, un quatrième et dernier motif nous apparaît légitimer une transformation du modèle pédagogique traditionnellement installé en enseignement supérieur. Le courant de la recherche collaborative (Desgagné, Bednarz, Couture, Poirier \& Lebuis, 2001) qui s'est progressivement développé a rendu compte de la fécondité d'un travail en collaboration entre universitaires et intervenants des milieux scolaires au profit de la coconstruction d'un savoir conjoint qui allie les savoirs conceptuels des premiers aux savoirs pratiques des seconds. Sans cantonner l'un et l'autre dernière une seule position de savoir, chacun en possède toutefois une dominante (Desgagné, Fortier \& Jobin, 2011) et l'établissement d'une collaboration à partir d'un objet de questionnement commun peut donner lieu à une source de développement professionnel à la fois pour l'un et l'autre acteur. Une conséquence de cet élément pour la formation continue peut impliquer qu'il n'y ait pas que les étudiants qui soient délibérément en situation d'apprentissage (Bereiter \& Scardamalia, 1989), mais aussi le professeur. Si ce dernier conserve l'essentiel de la responsabilité du volet de formation, il est dans son intérêt d'offrir un espace au savoir d'expérience que les étudiants — des enseignants d'expérience dans le cas de plusieurs programmes de formation continue en éducation - pourront partager. Un tel souci favorisera un mutualisme entre les savoirs théoriques et pratiques.

Sur la base de tels constats, nous avons participé à l'élaboration d'un programme de formation $^{2}$ (Allaire, Pellerin, Beaudoin, Couture \& Turcotte, 2010) dont le fonctionnement vise à promouvoir une métaphore de l'apprentissage qui soit davantage axée sur la participation (Sfard, 1998), voire la création (Pavoola, Lipponen \& Hakkarainen, 2004). Ce programme a pour principal objet d'apprentissage l'intervention en classe multiâge, dans un contexte de classe en réseau, et il est d'abord destiné aux enseignants en exercice. Dans les pages qui suivent, nous exposons le cadre de référence ayant présidé au modèle pédagogique du programme, nous en présentons le fonctionnement puis nous exposons des retombées issues des processus de participation et de création qu'il a cherché à soutenir auprès d'étudiants ayant pris part à un

2 Il s'agit d'un programme de formation dispensé en réseau par l'Université du Québec à Chicoutimi (UQAC), l'Université du Québec en Abitibi-Témiscamingue (UQAT) et l'Université du Québec en Outaouais (UQO). 
cours qui en fait partie. Les résultats qui seront présentés sont issus des questions de recherche suivantes :

- Comment les interactions sociales s'organisent-elles entre les participants au sein du programme de formation?

- Comment les participants contribuent-ils au processus de construction de connaissances?

- Quel est le contenu qui résulte de ce processus de construction?

Par ces questions, nous souhaitions apporter un éclairage au développement pédagogique en enseignement supérieur ; un créneau encore peu étudié (Endrizzi, 2011).

\section{Cadre de référence du programme de formation continue}

Les enseignants en classe multiâge ${ }^{3}$ se retrouvant souvent dans de petites écoles, là où il $\mathrm{y}$ a peu d'élèves, et donc peu de collègues avec qui entretenir une collaboration professionnelle, une modalité stratégique importante du programme de formation étudié ici consistait à permettre aux étudiants d'intégrer une communauté d'enseignants qui travaillent en réseau dans leur classe multiâge, et d'y faire une contribution. Par conséquent, un cadre de référence centré sur l'amélioration collective d'idées (Bereiter \& Scardamalia, 2003) a été adopté pour aiguiller le modèle pédagogique mis en avant. Plus spécifiquement, nous nous sommes inspirés des 12 principes de coélaboration de connaissances (knowledge building), initialement formulés par Scardamalia et Bereiter (2004), que nous avons remaniés en cinq (Allaire \& Lusignan, 2011). Le déroulement général du programme de formation s'en inspire. Voici une brève description de chacun de ces principes.

\section{Collaboration et complémentarité des idées à partir de questions réelles et de problèmes authentiques}

Le questionnement est considéré comme un élément fondamental de toute entreprise d'apprentissage. Plutôt que de fournir d'entrée de jeu un contenu bien ficelé, le professeur cherche d'abord à mettre à contribution le questionnement des étudiants en regard du contexte de formation. En l'occurrence, il s'agit d'identifier conjointement avec eux des questions importantes qui prévalent actuellement dans le monde de l'éducation en ce qui a trait à l'intervention en classe multiâge. Les questions que les étudiants se posent et les problèmes qu'ils désirent résoudre créent un "vide à combler» qui est propice à l'apprentissage. En d'autres mots, ils créent un besoin d'apprendre, de découvrir de nouvelles choses. L'objectif ici est de parvenir à un engagement collectif, de développer un esprit d'équipe et de faire prendre conscience aux étudiants que tous les participants du cours cheminent ensemble.

\section{Amélioration et diversification des idées de manière participative par le biais du discours}

À ce niveau, il s'agit pour le responsable de la formation d'encourager les étudiants à exprimer les idées qu'ils ont par rapport aux questionnements identifiés par le groupe. L'amélioration des idées étant un processus progressif, des moyens, par exemple la rédaction de notes sur un forum électronique, sont utilisés pour en conserver des traces écrites. Cela permet plus aisément de retourner aux idées antérieures pour les consulter de nouveau, les revisiter, les bonifier, etc. Ainsi, contrairement à ce qui se passe la plupart du temps dans une classe traditionnelle, l'enseignant implique de près les étudiants dans une démarche d'investigation

\footnotetext{
${ }^{3} \mathrm{Au}$ Québec, le cheminement scolaire au primaire s'effectue à travers un continuum de trois cycles d'apprentissage, chacun d'eux renfermant deux années. Le premier cycle regroupe la première et la deuxième années, et ainsi de suite. Une classe est dite multiâge lorsqu'elle est composée d'élèves appartenant à au moins deux années. Une classe multiâge est dite intracycle lorsqu'elle est constituée d'élèves des années d'un même cycle (e.g. première et deuxième années). Elle est dite intercycle lorsqu'elle est constituée d'élèves de cycles différentes (e.g. quatrième et cinquième années). Les classes multiâges intercycles sont généralement reconnues comme étant plus complexes à gérer.
} 
visant à mieux comprendre les questions importantes en lien avec l'objet du cours. Il n'est donc pas le seul à y contribuer; les participants le font aussi, notamment par l'explicitation de leurs savoirs d'expérience et la mise en relation de celui-ci avec des écrits de nature conceptuelle.

\section{Responsabilisation des étudiants dans un climat démocratique}

La classe universitaire qui travaille selon une approche d'amélioration collective des idées se partage des responsabilités. Tous sont impliqués dans l'identification de ces responsabilités; et non pas seulement le professeur. Cela implique qu'il y ait des moments où le travail s'effectue de façon individuelle, en équipe, en grand groupe. En outre, il y a une diversité de tâches réparties entre les étudiants, en fonction de leurs forces, de leurs intérêts, de leur contexte de pratique. Ce qui est visé au niveau de ce principe, c'est que chacun ait sa part de responsabilité et qu'il participe à l'effort collectif d'avancement de la compréhension.

\section{Considération de sources fiables tout au long de la démarche d'investigation}

Dans une classe universitaire qui valorise l'amélioration des idées et pas seulement une métaphore de l'appropriation du savoir existant, les contenus tels qu'on les connaît occupent toujours une place importante. Elle est toutefois différente. Plutôt qu'être des objets d'appropriation en soi, les contenus sont utilisés pour éclairer la démarche de compréhension du groupe. En outre, ces contenus peuvent aussi être discutés, questionnés, voire remis en question, sur la base d'arguments étayés par les participants.

\section{Évaluation partagée, en contexte, tout au long du processus}

Enfin, une place importante est accordée à l'observation des processus sociaux et cognitifs en cours de processus (Nirula, Woodruff, Scardamalia \& MacDonald, 2003). Cette observation est ancrée à même la démarche d'amélioration des idées à laquelle la classe prend part, plutôt qu'être effectuée en vase clos, c'est-à-dire dans un contexte distinct ou dans le cadre d'une activité indépendante. Plus concrètement, cela signifie que les étudiants sont constamment en alerte par rapport à la démarche d'investigation à laquelle ils prennent part. Ils réfléchissent à la pertinence des initiatives qui sont prises et des contributions qui sont effectuées, en cours de démarche, et pas seulement a posteriori. Il s'agit donc de prévoir des moments de régulation périodiques pour faire le point sur la qualité et la pertinence du travail accompli jusque-là. L'idée générale de ce principe est donc d'amener les étudiants à prendre du recul et à faire preuve de rigueur par rapport au travail effectué, plutôt que de dépendre uniquement des rétroactions et de la sanction de l'enseignant.

\section{Mise en place du programme de formation}

Le programme de formation que nous utilisons pour illustrer le modèle pédagogique qui vient d'être présenté porte sur l'intervention dans les petites écoles et les classes multiâges en réseau. Il comporte trois cours; chacun d'eux étant sous la responsabilité d'une des trois universités partenaires (UQAC, UQAT, UQO). En l'occurrence, lorsque l'UQAC dispense le cours dont elle est responsable, les étudiants admis dans les deux autres universités sont accompagnés par le professeur de l'UQAC, et ainsi de suite. Puisqu'une partie importante des étudiants visés par ce programme de formation est susceptible d'être située en milieu rural, et donc éloignée d'un centre universitaire, les cours sont dispensés en réseau par l'entremise d'un forum électronique (Knowledge Forum, KF) et d'un outil de vidéoconférence (VIA) accessible à partir d'un ordinateur.

Nous parlons de cours «en réseau » plutôt que de cours strictement «en ligne » puisque, bien qu'il n'y ait pas de rencontres en face à face à l'université, nous considérons néanmoins que l'environnement d'apprentissage en est un hybride, c'est-à-dire qui combine à la fois des interactions sociales en ligne et en présentiel (Laferrière, Lamon \& Breuleux, 2006). Or, dans notre contexte de formation, les interactions en face à face n'interviennent pas entre les étudiants et le responsable de cours, mais plutôt dans la classe multiâge même des étudiants qui, rappelons-le, sont pour la plupart des enseignants en exercice. Il s'agit du principal lieu duquel 
ils s'inspirent pour identifier des questionnements à aborder dans le cadre des interactions en ligne du cours, ce qui contribue à concrétiser le principe de «Collaboration et complémentarité des idées à partir de questions réelles et de problèmes authentiques » présenté à la section 2.1. En outre, la classe dans laquelle les étudiants enseignent est aussi le principal lieu de réinvestissement de ce qu'ils apprennent et développent dans le cours. Les cours du programme de formation entretiennent donc un lien étroit entre le contexte de pratique des enseignantsétudiants, soit leur principal lieu de participation, et les interactions qui se déroulent en ligne entre les étudiants et le professeur, lieu d'amélioration des idées, voire de création.

La métaphore de la participation prend aussi un sens additionnel et, pour l'illustrer, nous utilisons l'exemple d'un des trois cours du programme de formation: «Environnement collaboratif et apprentissage en réseau ». Dans ce cours, des modèles d'environnements axés sur la collaboration et l'apprentissage en réseau apportent un éclairage à la façon de gérer la complexité inhérente à la classe multiâge (Allaire, Hamel, Gaudreault-Perron \& Laferrière, 2012). Les étudiants sont amenés à élaborer un projet de mise en réseau de leur classe (Allaire \& Lusignan, 2011) afin d'intégrer la communauté panquébécoise de l'École éloignée en réseau (ÉÉR), un regroupement d'enseignants qui amènent leurs élèves à apprendre avec des élèves d'autres écoles, en étant reliés par des technologies de l'information et de la communication (TIC) (Laferrière, Hamel, Allaire, Turcotte, Breuleux, Beaudoin et al., 2011). Les enseignants du cours sont invités à positionner leurs questionnements à partir d'artefacts produits par cette communauté et à tenter de faire une contribution originale pouvant y être réinvestie. Ainsi, les interactions qui ont lieu dans le cadre du cours ne se déroulent pas en vase clos. Un effort d'arrimage est réalisé pour les ancrer dans des modèles et concepts théoriques ainsi que dans l'expérience de terrain des étudiants et l'activité d'une authentique communauté, l'ÉÉR.

Un tel mode de fonctionnement est possible grâce à la mise à contribution de TIC synchrones et asynchrones, qui ont un rôle de médiation crucial dans les interactions entre les individus dans notre contexte de formation. Hebdomadairement, les étudiants du cours se rencontrent quelques heures en vidéoconférence pour échanger à propos de divers questionnements soulevés en lien avec l'objet du cours. Ils s'y penchent sous l'éclairage de lectures préparatoires qui offrent une perspective conceptuelle, tout en étayant leurs propos de l'expérience vécue dans leur classe. Le responsable du cours s'occupe de l'animation des rencontres, il apporte des précisions quant à la compréhension des concepts et assure un rôle de médiation entre la théorie et la pratique. Entre ces rencontres, les participants au cours poursuivent leurs échanges, mais cette fois en asynchrone à l'aide du KF, un forum électronique dont l'accès est semi-public. Les idées élaborées par le groupe y sont conservées et peuvent donc être revisitées et améliorées à tout moment. Les traces que le forum permet de conserver peuvent aussi être réutilisées dans un cours ultérieur, voire par les étudiants d'une cohorte suivante. Cet aspect de partage des artefacts discursifs élaborés est discuté avec les étudiants, qui y voient généralement une authenticité supplémentaire au travail effectué dans le cadre du cours. L'intérêt est de situer les activités du cours par rapport à une certaine historicité qui est progressivement construite par une communauté partageant un but commun à un moment ou à un autre. C'est dans cette optique que les artefacts produits par la communauté de l'ÉÉR sont mis à contribution, qu'il s'agisse, par exemple, du guide pédagogique auquel des enseignants d'expérience ont participé (Allaire \& Lusignan, 2011), des exemples d'activités d'apprentissage en réseau disponibles, de témoignages audio et vidéo, des rapports de recherche disponibles sur son site $\mathrm{web}^{4}$, etc.

\section{Méthodologie et résultats}

Dans une perspective de recherche, nous nous sommes intéressés à la dynamique interactionnelle, en particulier celle dans le KF, qui a pris forme entre les participants du cours «Environnement collaboratif et apprentissage en réseau » qui s'est déroulé à l'hiver 2011. Sept étudiants en formation continue provenant de six régions administratives du Québec y ont

\footnotetext{
${ }^{4} \mathrm{http}: / /$ www.eer.qc.ca
} 
participé. Il y avait cinq femmes et deux hommes, et six étudiants sur sept étaient enseignants en exercice dans une classe multiâge. Quatre femmes enseignaient au primaire et les deux hommes enseignaient au secondaire. Au total, en incluant le responsable du cours, il y avait donc huit participants.

Des données de base concernant la participation au KF sont d'abord fournies. Elles proviennent des outils d'analyse intégrés au forum. Pour répondre au premier objectif, une analyse du réseau social au sein du KF a été effectuée à l'aide du logiciel NetDraw. Cette analyse a permis d'identifier qui est intervenu auprès de qui et selon quelle ampleur. Pour répondre au second objectif, des marqueurs de relation associés à la coélaboration de connaissances (marqueurs d'ajout, d'opposition, de confrontation, de nuance) ont été utilisés avec les outils de la trousse Calico. Cela a permis d'effectuer un premier repérage de passages textuels contribuant potentiellement à l'approfondissement du discours de groupe. Ensuite, une analyse de contenu a été effectuée par deux personnes à partir de ces passages afin de valider qu'ils traduisaient bien leur sens en contexte d'usage. Pour ce qui est du troisième objectif, nous avons demandé à un étudiant du cours et à une personne extérieure à celui-ci de rédiger ce qu'ils comprenaient du contenu d'une des sept principales thématiques développées sur le KF. De plus amples précisions méthodologiques sont fournies au moment de présenter les résultats de chaque objectif afin de faciliter la compréhension.

\section{Dans quelle mesure le KF a-t-il été utilisé?}

Pour l'ensemble du cours, qui s'est échelonné sur quelque 15 semaines, 197 contributions ( $\mathrm{x}=24.6)$ ont été écrites dans le KF. Par contributions, nous entendons des contenus issus, par exemple, de lectures, de situations vécues sur le terrain qui ont été échangés et discutés par les participants. Ce peut être aussi des appréciations plus personnelles à l'égard d'idées ou du travail effectué par un participant. Ce nombre inclut les contributions rédigées par le professeur responsable du cours, qui était aussi un participant actif aux échanges asynchrones. Plus précisément, 143 notes (messages) ont été rédigées par le groupe de participants. Parmi elles, $88 \%$ étaient des élaborations, c'est-à-dire des notes reliées à une note écrite antérieurement. Une élaboration témoigne, de la part du participant qui la rédige, d'une intention de poursuivre des idées existantes, ce qui s'inscrit dans l'optique d'un discours progressif (Bereiter \& Scardamalia, 1993). Par ailleurs, soulignons que la presque totalité des notes $(\mathrm{N}=142)$ ont un seul auteur. La seule note qui contient plusieurs auteurs reflète une propriété collective de son contenu.

Dans un autre ordre d'idées, le KF permet visuellement de suivre l'enchainement des échanges et interaction. Pour ce faire, il relie les élaborations par un trait et une flèche, tel qu'illustré à la Figure 1.

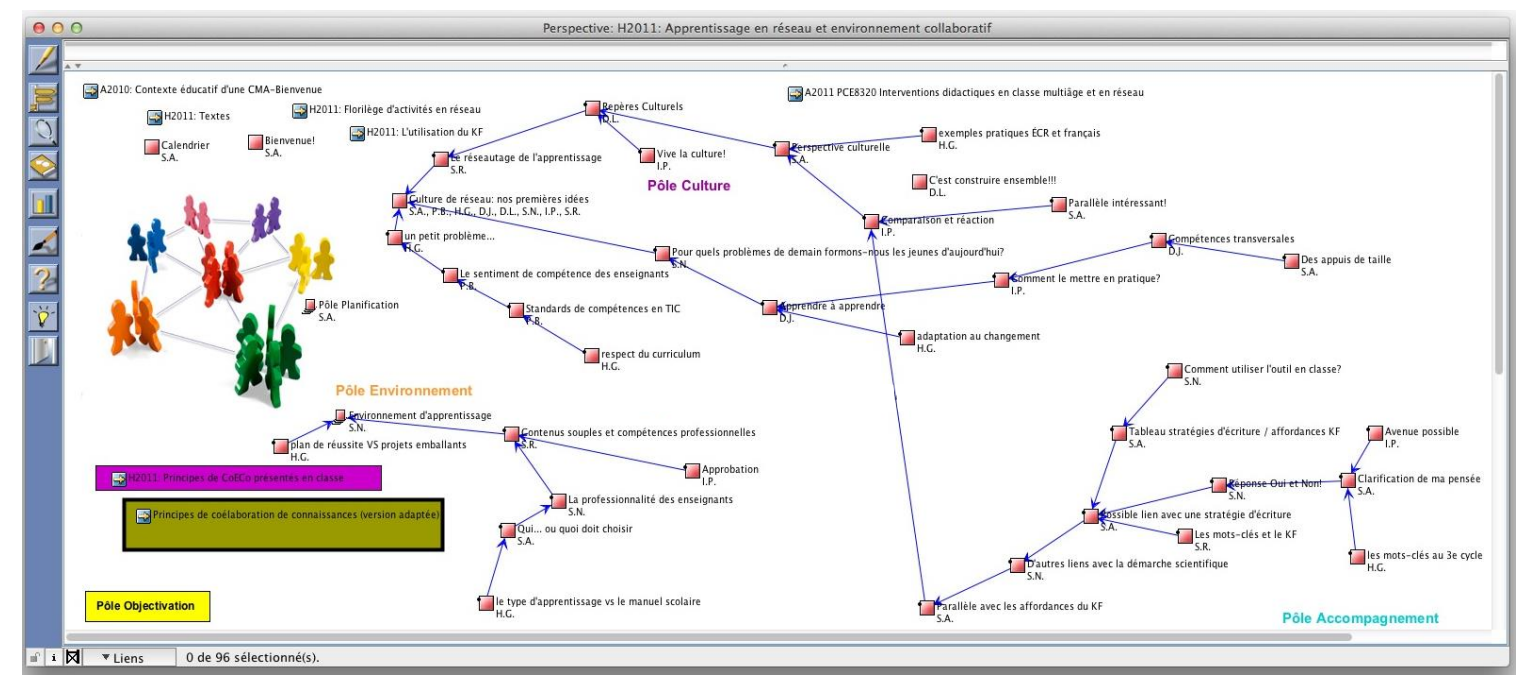

Fig. 1 Illustration de l'environnement Knowledge Forum 
Chaque boîte de couleur rouge représente une note. Lorsque deux notes sont reliées par un trait (couleur bleue), cela indique que la note qui pointe, par une tête de flèche, vers une autre note est élaborée à partir de cette dernière, donc cherche à poursuivre l'idée qu'elle renferme. Ainsi, en vertu du principe d'amélioration et diversification des idées, plus longs sont les enchainements de notes reliées par des flèches (appelées enfilades), plus cela devrait rendre compte d'un approfondissement du discours. Les enfilades présentes dans le cours qui fait l'objet de la présente analyse contiennent, en ordre croissant : 5, 6, 6, 7, 12, 20, 21 et 28 notes. Cela suggère un intérêt marqué pour les thématiques abordées, voire un souci important de la part des participants de s'arrimer aux idées qui précèdent afin de faire cheminer le discours collectif. Ce constat nous apparaît d'autant plus intéressant qu'il est généralement plus aisé de démarrer un nouveau sujet d'échanges que de chercher à en approfondir un existant, car cette dernière alternative requiert l'ajout d'éléments de contenus plus pointus.

En outre, parmi les 143 notes rédigées par les participants, deux étaient des notessynthèses, ou plus précisément des notes «Élever le propos ». Cela signifie que leur auteur a délibérément cherché à regrouper un ensemble de notes afin d'en tirer certains constats, de faire le point sur l'avancement des échanges de la communauté professionnelle. L'une a été écrite par une étudiante alors que l'autre a été écrite par le formateur.

Les 54 autres contributions sur les 197 au total représentent ce qu'on appelle sur le KF des annotations. Il s'agit de commentaires plus personnels qui s'adressent habituellement à un individu en particulier et qui est de nature socioaffective. L'objectif de ces commentaires ne consiste donc pas spécifiquement à approfondir les échanges.

Tableau 1 Données de base de la participation au Knowledge Forum.

\begin{tabular}{ccccc}
\hline $\begin{array}{c}\text { Contributions } \\
\text { totales }\end{array}$ & Nouvelles notes & Élaborations & $\begin{array}{c}\text { Notes «Élever } \\
\text { le propos } »\end{array}$ & Annotations \\
\hline 197 & 15 & 126 & 2 & 54 \\
\hline
\end{tabular}

Aussi, lors de la rédaction d'une note dans le KF, il est possible d'effectuer des références à des propos écrits antérieurement par d'autres participants ou soi-même. Cette possibilité s'apparente aux citations que l'on retrouve dans les écrits scientifiques. Les données du cours étudié dans le cadre de cet article indiquent que les participants ont effectué 121 références, ce qui nous apparaît être un indice supplémentaire illustrant le souci des participants de tenir compte de l'historique des échanges.

Nous présentons maintenant les résultats spécifiques à chacune des principales questions de recherche ciblées. Les résultats sont précédés de l'explication de la méthode d'analyse utilisée pour les obtenir. Nous avons procédé ainsi, plutôt qu'à partir d'une seule section regroupant la totalité des procédures utilisées, puisque nous jugeons que cela permettrait de mieux comprendre, en contexte de lecture, de quelle façon les analyses ont été menées pour chaque question.

Comment les interactions sociales s'organisent-elles entre les participants au sein du programme de formation?

Dans le champ de l'apprentissage collaboratif supporté par l'ordinateur (ComputerSupported Collaborative Learning, CSCL), l'analyse de réseaux sociaux (social network analysis) (Degenne \& Forsé, 1994; Wasserman \& Faust, 1994) est de plus en plus reconnue comme un moyen efficace permettant de représenter la structure des interactions dans une communauté d'apprenants qui travaillent en ligne à partir d'outils asynchrones (Haythornwaite \& De Laat, 2010; Marcos-Garcia, Martínez, Dimitriadis \& Anguita, 2007). Baker-Doyle et Yoon (2011) et Penuel, Riel, Krause et Frank (2009) l'utilisent aussi pour représenter les relations au sein de réseaux professionnels d'enseignants. Reffay et Martínez-Monés (2011) définissent l'analyse de réseaux sociaux ainsi: « Social Network Analysis (SNA) is an approach to analysis that focuses on the study of the relationships among members in a community or a group. It affords alternative ways of studying collaboration, in a way that is both formal and intuitive. [...] 
A classical starting point for a study based on SNA is the construction of a sociometric graph defining relationships between individuals. Depending on whether the relation is directed or not, the resulting graph can be either a digraph (i.e. oriented/directed graph) or an undirected graph ».

$\mathrm{Au}$ sein d'une communauté d'élaboration de connaissances, deux relations importantes à considérer sont celles de la lecture et de la réponse aux écrits d'autrui puisqu'elles sont au cœur de ce qui s'y effectuent et de la progression d'un discours collectif (Bereiter \& Scardamalia, 1993). C'est donc à ces relations que nous nous sommes principalement intéressés pour observer l'organisation des interactions entre les participants à partir de ce qu'ils ont écrit dans le KF, traces dont nous avons présenté les données quantitatives de base dans la section qui précède. Pour ce faire, nous avons utilisé le progiciel NetDraw, spécialement conçu pour le traçage de sociogrammes. Un sociogramme est « un graphe dont les sommets représentent des acteurs et les arcs, des relations entre ces acteurs ». Pour donner un exemple de ce qu'il permet d'illustrer, nous en avons tracé un (Figure 2) inspiré par des études qui rendent compte des interactions qui ont lieu dans une situation où le modèle pédagogique en est un essentiellement d'acquisition par la voie d'un enseignement frontal (Cazden, 1988, 2001; Flanders, 1970; Sinclair \& Coulthard, 1975), tel qu'on en rencontre en enseignement supérieur.

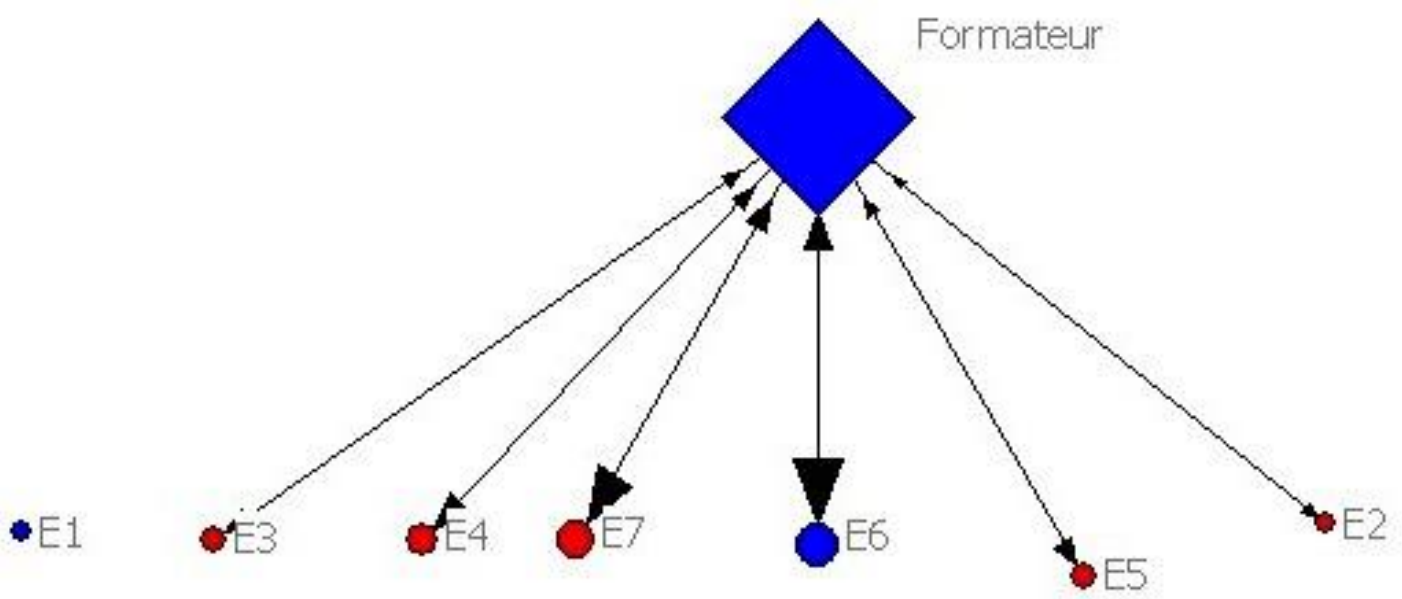

Fig. 2 Sociogramme qui représente les interactions dans une classe dont le modèle pédagogique dominant est l'enseignement magistral

La taille des nœuds (cercles et losange) représente le temps de parole des individus (formateur et étudiants E1 à E7) alors que les arcs (traits) et leurs têtes de flèches (leur envergure) rendent compte des interactions de type «question - réponse - rétroaction » entre les acteurs. Une telle figure permet de dégager deux principaux constats. D'une part, c'est essentiellement le formateur qui exprime quelque chose et, d'autre part, il n'y a pas d'interactions directes entre les étudiants.

Les éléments de base d'un sociogramme étant illustrés, nous présentons maintenant les résultats issus des analyses effectuées à partir des données du cours étudié dans le cadre de cet article. La Figure 3 est un sociogramme où la taille des nœuds est proportionnelle au nombre de contributions (notes et annotations) rédigées sur le KF par les participants. La taille des têtes de flèches (de A vers B), c'est-à-dire leur envergure, est quant à elle proportionnelle au nombre de notes de B lues par A. Deux couleurs ont été utilisées pour distinguer le sexe des participants : le bleu pour les hommes et le rouge pour les femmes. La forme différencie le formateur (carré) des étudiants (cercles). 


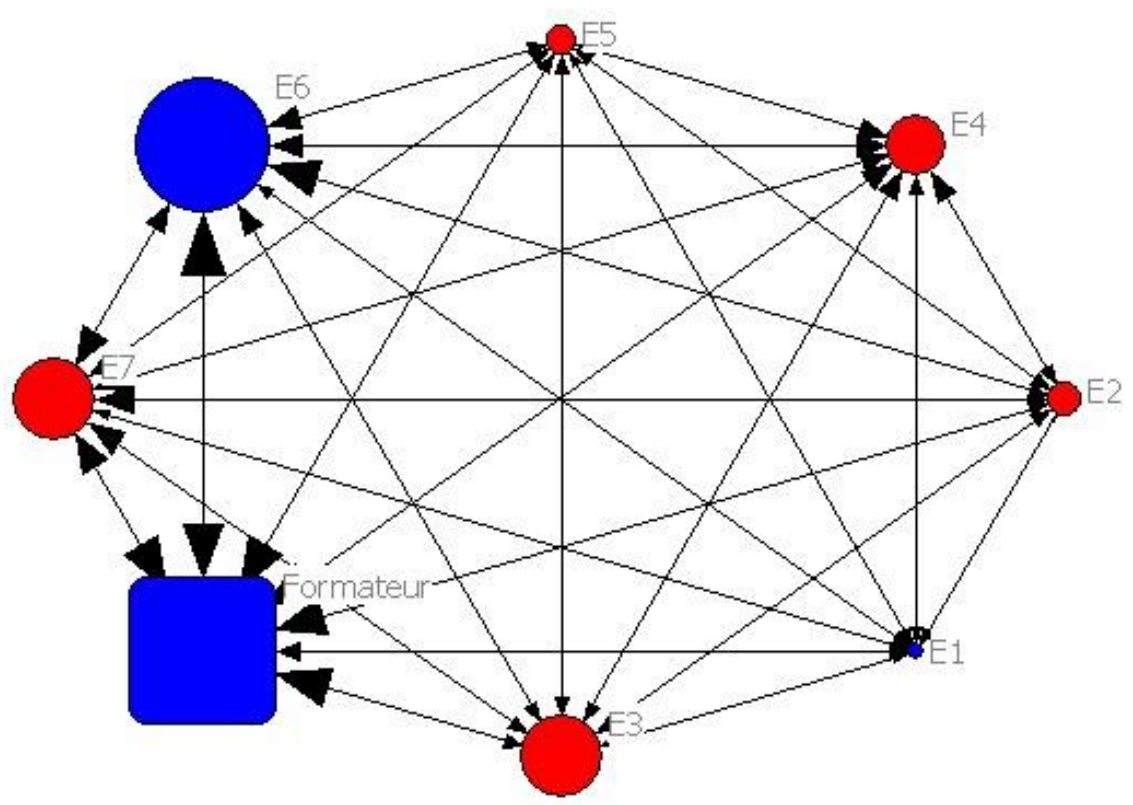

Fig. 3 Contributions écrites et relations de lecture entre les participants au cours

Globalement, un constat marquant se dégage, en comparaison avec le sociogramme de la Figure 2 qui rendait compte de ce qui se déroule au plan interactionnel en situation où l'enseignement frontal a prédominance. Ici, l'essentiel du contenu qui a transité dans le cadre du cours n'est pas provenu que du formateur; les étudiants y ont contribué de façon importante, bien que ce soit dans des proportions différentes. En outre, de façon plus spécifique, on peut remarquer que la production de E6 est proche de celle du formateur, tandis que les notes du formateur sont davantage lues par l'ensemble des participants. Malgré cela, une majorité de participants a lu des notes des autres, ce qui rend compte d'un engagement dans la tâche. On peut aussi constater que E1 ne lit que peu de contributions des autres participants. Il en va de même pour la rédaction de notes qu'il effectue. Dans cette représentation graphique, les types de contributions (annotations, notes, notes-synthèses) ont été considérés comme équivalentes du point de vue de leur importance pour la progression du discours de la communauté.

Le sociogramme qui suit (Figure 4) représente l'implication en écriture et les relations d'écriture avec les notes des autres participants. En d'autres mots, on peut y observer qui rédige le plus et qui le fait à partir des contributions de qui. Ici, puisque nous représentons uniquement des données qui concernent la rédaction, nous avons pondéré chaque type de contribution (Annotation : une, Notes : quatre, Notes-synthèses : six) pour tenir compte du fait que toutes les contributions n'impliquent pas la même exigence sur le plan cognitif et qu'elles ne contribuent pas de la même façon à la progression du discours collectif. Dans ce sociogramme, une relation de $\mathrm{A}$ vers $\mathrm{B}$ représente le degré d'implication des écrits de A vis-à-vis des contributions de B. Il modélise donc, d'une certaine manière, 1) le degré d'implication en écriture (par la taille du nœud), 2) comment le participant répartit son attention en écriture vis-à-vis des autres participants (flèches partant d'un nœud) et 3) quelle attention en écriture il reçoit des autres (flèches convergeant vers lui). Nous avons choisi de tracer en lignes fines et grises les relations de plus faible intensité (inférieure à $10 \%$ de la valeur maximale d'implication calculée) et en traits gras et noirs celles qui sont plus intenses. 


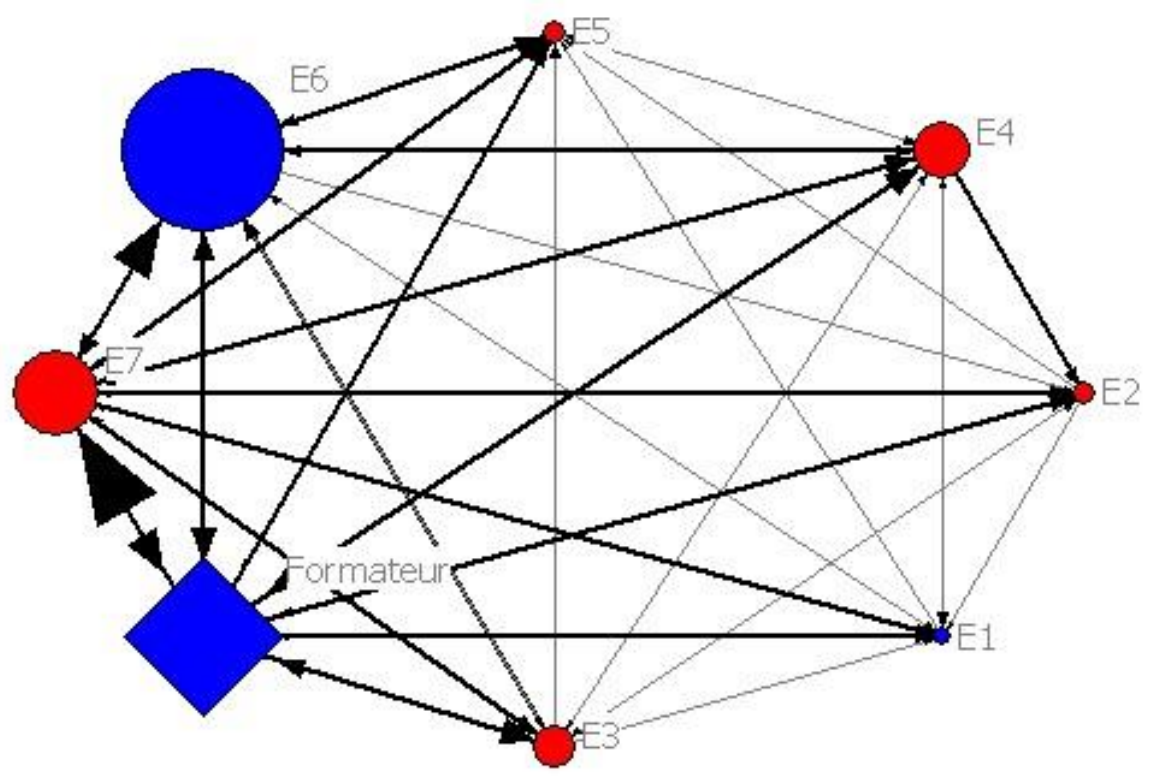

Fig. 4 Implication en écriture et relations d'écriture

Au premier regard, ce qui est marquant, c'est la présence d'interactions entre plusieurs participants du cours, c'est-à-dire qu'ici, plusieurs utilisent les contenus des uns et des autres pour y ajouter leurs idées propres. On remarque aussi que des participants semblent préférer utiliser les contributions de certains pour ajouter les leurs. Parmi les constats plus pointus, on remarque que l'implication de E6 en rédaction est supérieure à celle du formateur. On remarque aussi que l'attention portée à E7 par les autres étudiants est assez faible, comparativement à l'utilisation que le formateur fait de ses contributions, ainsi qu'à l'utilisation que E7 effectue de celles des autres participants. Enfin, alors que l'implication de E6 est la plus élevée, on note que son attention envers les autres est plutôt faible. Cette particularité s'explique par le fait qu'à la différence des autres étudiants, il a réalisé son travail de bilan individuel de la mise en œuvre d'une activité d'apprentissage en réseau dans le KF, produisant ainsi de nombreuses notes en réponse à ses propres notes.

\section{Comment les participants contribuent-ils au processus de construction de connaissances?}

La dialectique de Popper et Eccles (1977) étant un ancrage théorique de la communauté d'élaboration de connaissances, nous avons observé la présence d'idées qui rendent compte d'ajouts (thèse), mais aussi d'oppositions, de confrontations, de nuances (antithèse), etc. à travers les contributions des participants. C'est à travers les allers-retours entre de tels types d'idées qu'on peut voir se dégager, progressivement, un nouvel état de compréhension, de connaissance (synthèse).

Sur le plan méthodologique, nous avons utilisé des groupes de marqueurs de liaison pour identifier la présence d'ajouts et d'éléments d'antithèse dans le discours des participants. D'abord, nous avons établi deux listes de marqueurs (ajout ; opposition, confrontation et nuance) à partir de travaux effectués par le Centre de rédaction de l'Université d'Ottawa, mais aussi en consultant le discours écrit dans le KF afin d'enrichir les deux listes initiales. Voici quelques exemples de marqueurs pour chaque type.

- Ajout : ainsi que, par surcroit, également, en outre, en plus, j'ajouterais que

- Opposition, confrontation et nuance: cependant, contrairement à, en revanche, par contre 
Dans un second temps, un plugiciel d'analyse lexicale, développé par l'équipe Afford@ ance ${ }^{5}$ de 1'UQAC, a été utilisé pour dénombrer la présence des marqueurs. Cet outil est accessible à même le KF. Quelque 834 marqueurs d'ajout et 275 marqueurs d'opposition, de confrontation ou de nuance ont été répertoriés dans l'ensemble des notes écrites par les participants du cours.

Une telle analyse ne fournissant aucune indication permettant de savoir si l'emploi des marqueurs introduisait réellement un contenu de la nature qu'il induit, une analyse supplémentaire a ensuite été effectuée. La totalité du discours élaboré dans le KF dans le cadre du cours a été importée dans les outils d'analyse Calico, développés par le laboratoire de recherche Science Techniques Éducation Formation (STEF) ${ }^{6}$ de l'ENS de Cachan et par le Groupe de recherche en informatique, image, automatique et instrumentation (GREYC) ${ }^{7}$ de l'Université de Caen. En particulier, les outils Bobinette et Show Forum, qui permettent de représenter le déroulement temporel des fils d'échanges (enfilades) ainsi que le suivi de thématiques prédéfinies, ont été utilisés pour identifier, à travers les échanges du forum, les marqueurs de liaison des deux groupes définis. Un avantage de ces outils est qu'ils permettent de visualiser clairement les marqueurs dans leur contexte d'utilisation réelle. La Figure 5 fournit un exemple de visualisation.

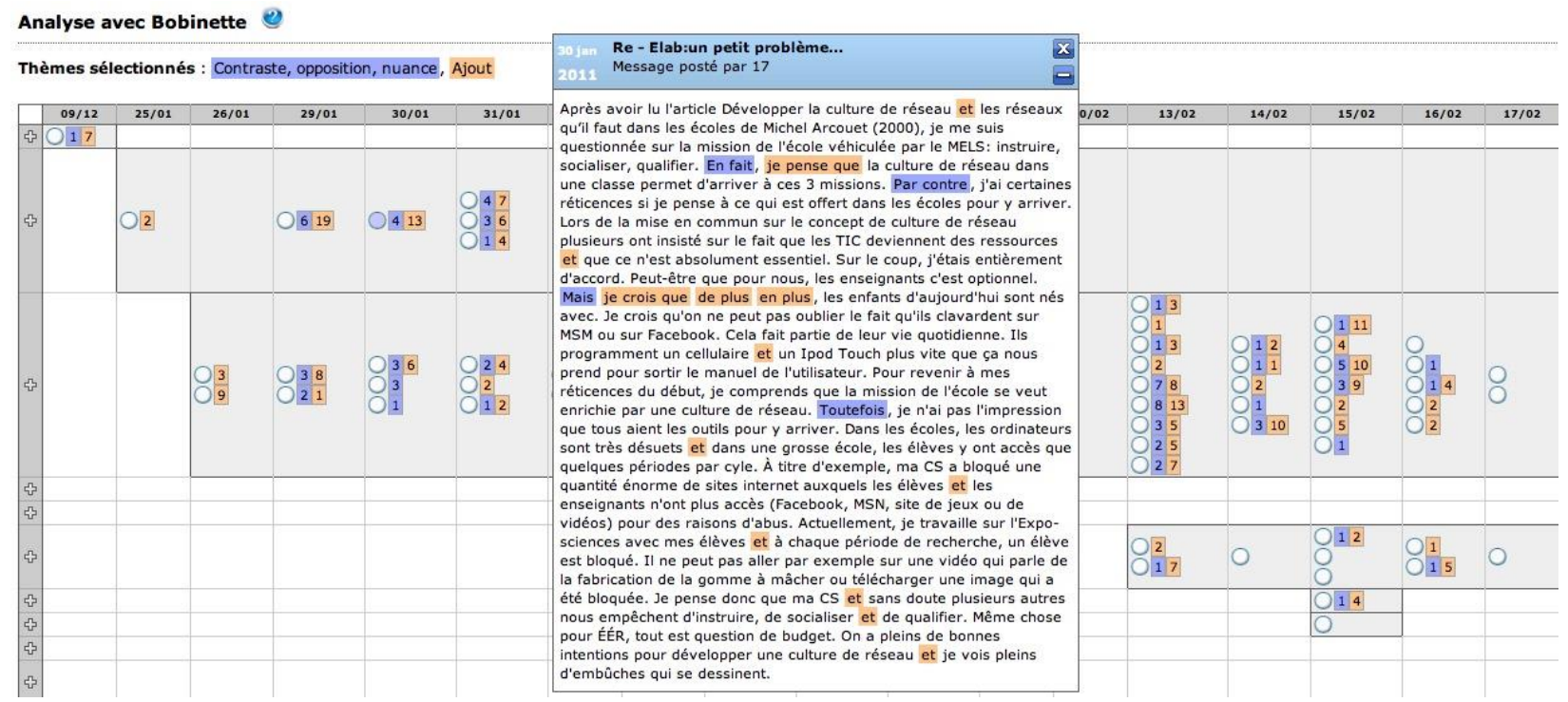

Fig. 5 Illustration des outils d'analyse Bobinette et ShowForum

Une vérification de la correspondance des marqueurs appartenant aux deux groupes retenus a été effectuée pour environ $50 \%$ du contenu provenant du forum du cours. Au final, à la suite d'un accord interjuge mené par deux personnes qui a engendré des ajustements dans la manière de procéder à cette analyse, il a pu être établi que, dans environ $75 \%$ des cas, l'emploi d'un marqueur de type «Ajout» introduisait réellement un contenu de cette nature. C'est donc dire qu'une seule analyse automatisée de ce type de marqueur, sans vérifier le contexte d'usage, permet de révéler trois fois sur quatre que le marqueur employé rend bien compte d'un ajout de contenu. Les marqueurs qui ont parfois dû être écartés, faute de contribuer à un réel ajout, sont les suivants : et, de plus, en plus, aussi, puis, ensuite, ainsi que, également, outre. Pour ce qui est de l'emploi des marqueurs de type «Opposition, contraste et nuance», il s'est révélé concordant dans plus de $80 \%$ des cas. Les marqueurs qui ont parfois dû être écartés sont les suivants : cependant, mais, seulement, alors que.

À l'issue de cette séquence d'analyse, à partir des 834 marqueurs d'ajout et des 275 marqueurs d'opposition, de contraste et de nuance, on peut extrapoler qu'à travers l'ensemble

\footnotetext{
${ }^{5} \mathrm{http}: / /$ affordance.uqac.ca

${ }^{6} \mathrm{http}: / /$ www.stef.ens-cachan.fr/

${ }^{7} \mathrm{https}$ ://www.greyc.fr/
} 
des notes écrites par la communauté, 626 ajouts de contenus, d'informations ont été effectués, ainsi que 220 assertions visant l'exploration contradictoire de propos énoncés. Cela correspond à 3,3 ajouts et à 1,2 opposition, contraste ou nuance par note ou annotation. De tels résultats permettent de constater que les interactions qui ont pris forme entre les participants de la communauté ont été mises au profit du partage d'une multitude d'idées.

\section{Quel est le contenu qui résulte du processus de construction de connaissances?}

Tout au long du cours, ce sont huit pôles ou thématiques qui ont émergé et qui ont été développés, de façon concomitante, relativement à son objet général, soit les environnements collaboratifs et l'apprentissage en réseau. Nous les énonçons ci-après, accompagnés d'une description qui rend compte sommairement du sujet de leurs échanges.

- Pôle «Environnement»: Définition et caractéristiques d'un environnement d'apprentissage en réseau.

- Pôle «Planification»: Comment s'y prendre pour élaborer une activité d'apprentissage en réseau?

- Pôle «Culture » : Caractéristiques de la culture de réseau et façons d'ancrer les apprentissages à effectuer par les élèves dans la perspective historique et culturelle du Programme de formation de l'école québécoise.

- Pôle «Accompagnement et intervention »: Rôles de l'enseignant dans une classe en réseau.

- Pôle «Technologie»: Enjeux technologiques inhérents au travail et à l'apprentissage en réseau.

- Pôle «Organisation»: Façons d'organiser le travail, de gérer la classe en situation de travail en réseau.

- Pôle «Objectivation»: Bilan des apprentissages effectués par les étudiants du cours.

Une approche dialectique étant appelée à se concrétiser en synthèse des éléments qui ont fait l'objet d'interactions, nous avons procédé ainsi pour rendre compte de la construction issue du travail de la communauté d'élaboration de connaissances dans le cadre du cours. Pour ce faire, deux manières différentes ont été utilisées. D'une part, une personne extérieure au cours et non experte du sujet a été sollicitée pour, d'abord, consulter les échanges de la communauté ayant trait au pôle «Culture» et, ensuite, en rendre compte dans ses propres mots (Tableau 2). D'autre part, nous présentons une seconde synthèse, celle du pôle «Environnement », qui a été rédigée par une étudiante du cours et qui avait participé activement aux échanges (Tableau 3). Chaque synthèse est d'une longueur approximative d'une page.

Tableau 2 Synthèse des éléments du pôle «Culture » (par une personne extérieure au cours)

Dans ce pôle, on a traité de culture de réseau, mais également à quelques reprises de culture au sens plus large de la définition de ce mot.

Les échanges ont été lancés par une mise en commun de ce que chacun entendait initialement par culture de réseau. L'aspect de nouveauté par rapport au passé en ressort ; on note également que celle-ci existe déjà dans la classe en dehors de toute technologie. La nature «sociale » du réseau est identifiée, bien que celui-ci ne se limite pas à des groupes sociaux comme Facebook. Son utilité en application technologique est aussi soulignée puisque le réseau offre la possibilité de décloisonner les matières, d'agir à titre de ressource de connaissances et de savoir-faire, en plus d'élargir les horizons tant des élèves que des enseignants. Enfin, le réseau et sa culture sont un prolongement des groupes de pratique réflexive.

Les étudiants du cours soulignent que la culture de réseau permet d'atteindre les trois objectifs du MELS $^{8}$ qui sont d'instruire, de socialiser et de qualifier, bien que les moyens mis à la disposition des

\footnotetext{
${ }^{8}$ Ministère de l’Éducation, du Loisir et du Sport.
} 
enseignants et des élèves pour y arriver soient parfois insuffisants. Les participants traitent des limites qu'on impose quant à l'accès à la connaissance sur Internet au nom de la moralité et de l'efficacité. Qui plus est, on y parle du fait que les jeunes apprenants sont souvent plus à l'aise dans cette culture électronique [numérique] que leurs enseignants, ce qui peut être un frein pour ceux et celles d'entre eux qui n'ont pas atteint une assez grande «littératie technologique », premier critère de compétence des TIC identifié par l'UNESCO. L'approfondissement de la connaissance et la création de connaissances sont les deux autres balises dont parle cette organisation internationale. D'ailleurs, on note dans les échanges une similitude importante entre ce qu'énoncent à ce sujet l'UNESCO et les travaux des auteurs Bereiter et Scardamalia, en y apportant toutefois davantage de précisions. Un problème important souligné par l'un des participants concerne la façon d'équilibrer les exigences individuelles des apprenants et celles du curriculum scolaire, tout en utilisant ces nouvelles technologies de mise en réseau. On extrapole que la difficulté sera d'ailleurs bientôt multipliée en raison de la nouvelle pondération de l'année scolaire à chacune des étapes et le retour au bulletin " chiffré ». Par contre, on reconnaît aussi que la culture de réseau permet d'exercer plus facilement une forme de démocratie à l'intérieur de la classe, en plus d'élargir la communauté scolaire au-delà des limites des murs de la classe. Puis, la qualité de la dynamique de groupe en tant qu'élément favorisant la culture de réseau est aussi citée.

Les échanges relatent aussi l'importance d'expliquer et de montrer aux élèves comment « apprendre à apprendre » plutôt que de leur enseigner un ensemble de connaissances dont certaines seront rapidement désuètes. Cela se traduirait notamment à travers les compétences transversales du Programme de formation de l'école québécoise (PFÉQ). On fait aussi remarquer que des orientations de l'UNESCO abondent dans un sens similaire.

Puis, on énonce l'importance des liens qui sont tissés entre les connaissances à l'ère d'Internet, appuyé par les propos de Sawyer. On discute de comment la connaissance s'articule par rapport à elle-même (i.e. du constructivisme), ou en s'ajoutant à la connaissance préalable de l'individu et du groupe (i.e. du socioconstructivisme). Des participants au forum fournissent des exemples en musique et en français à ce propos. On dit que la dynamique de groupe permet à l'apprenant de donner un sens supplémentaire à la connaissance et à mieux la mémoriser, en plus de faciliter ce processus de rattachement aux connaissances antérieures. Un participant illustre le propos en fournissant un exemple de comment il a pu parvenir à expliquer un concept difficile en physique en procédant sur la base de connaissances antérieures. Quelqu'un d'autre renchérit en expliquant pourquoi il est préférable de situer la connaissance dans un contexte social, historique et culturel. Puis, on fait un parallèle entre cette démarche et la méthode scientifique. Une autre personne fait le rapprochement entre l'utilisation des mots-clés sur KF et la démarche scientifique, une idée qui donne lieu à un échange à partir d'un tableau de classification des affordances de KF en regard de diverses stratégies d'apprentissage.

Finalement, on rappelle que ce n'est en n'ayant pas peur de confronter ses idées à celles des autres qu'on peut justement les arrimer les unes aux autres et qu'il faut éviter de toujours donner de la connaissance toute faite aux élèves

Tableau 3 Synthèse des éléments du pôle «Environnement» (par une étudiante du cours)

Cette note synthèse vise à rassembler nos premières idées relativement au concept d'environnement d'apprentissage. À ce propos, nous avons commencé par nous questionner sur le sens du concept. Qu'évoque-t-il ? Est-ce un concept porteur à partir duquel construire de nouvelles façons de faire pour nos classes? Ma synthèse se divise en quatre parties : 1) Définition ; 2) Ressources de l'environnement d'apprentissage ; 3) Rôles de l'enseignant ; 4) Ouverture et prospective.

\section{1) Définition}

- À partir des définitions consultées à travers les lectures, l'environnement d'apprentissage se définit ainsi pour nous : «Ensemble d'éléments (personnes, objets, conditions) qui créent un contexte permettant l'apprentissage à un groupe d'individus en établissant des relations d'interdépendance.

- Il est important de mentionner que trois auteurs parmi nous soulignent que ce concept leur 
était initialement inconnu sur le plan théorique.

- Aussi, une distinction s'établit avec le climat de classe et l'environnement d'apprentissage. «Pour créer un environnement d'apprentissage sain et émancipatoire, il faut un bon climat de classe », a dit quelqu'un, et ce climat est à mettre en place dès le début de l'année. En ce sens, on peut penser que le climat de classe fait référence à la gestion des comportements et aux routines établies.

2) Ressources de l'environnement d'apprentissage

En lien avec notre définition, les principales idées que nous avons trouvées sont les suivantes.

- Personnes : élèves, enseignant titulaire, autres enseignants, direction, service de garde, parents, famille, etc.

- Objets : matériel et artefacts variés, environnement physique et en ligne, activités d'apprentissage vécues.

- Conditions : climat de classe accessible et motivant, collaboration, orientations de l'école (projet éducatif), accessibilité des personnes et des objets, planification soutenue, communication entre les acteurs.

3) Rôles de l'enseignant

- L'enseignante joue un rôle important dans la création de l'environnement d'apprentissage. En effet, elle doit agir à titre de guide et d'aidant à l'apprentissage, mais aussi avec la participation de tous les apprenants. À ce sujet, la métaphore partagée par l'un d'entre nous appuie les propos que nous dégageons : "Chaque élève est capitaine de son bateau. Il navigue sur les eaux du savoir et il pêche où bon lui semble. Parfois, il fera une pêche miraculeuse, parfois il reviendra bredouille, parfois il se perdra. Mon rôle, c'est d'être un phare, un GPS, un opérateur radio et parfois... une équipe de sauvetage. »

- Ainsi, le rôle de l'enseignant dans un environnement d'apprentissage est de laisser de la place aux élèves en créant des contextes d'apprentissage authentiques qui font appel à leurs intérêts et favorisant un questionnement réel tout en s'appuyant sur une pratique réflexive soutenue. L'enseignante planifie, organise et gère l'environnement d'apprentissage en favorisant une responsabilisation et une collaboration entre tous les élèves.

4) Ouverture et prospective

- Pour terminer, quelqu'un nous a évoqué les possibilités d'ouverture interculturelle pour la classe du Knowledge Building International Project (KBIP) qui est un projet de collaboration internationale amenant des classes du Québec, de la Catalogne, du Mexique et de Hong Kong à travailler ensemble autour de diverses questions environnementales.

- Au moins une question demeure encore sans réponse claire à propos de notre pôle «Environnement »: La reddition de compte n'a-t-elle pas pour effet de standardiser les contenus et les moyens d'apprentissage dans l'environnement?

Bien que la façon de présenter ces résultats ne soit pas issue d'une analyse effectuée à partir de principes systématiques qui caractérisent des analyses qualitatives plus classiques, elle nous est apparue pertinente pour permettre au lecteur de constater le potentiel d'apprentissage, d'appropriation personnelle et de rétention pouvant résulter du modèle pédagogique mis en place.

\section{Discussion}

Les résultats de cette étude ciblée mettent en exergue un certain nombre de constats à propos de la dynamique interactionnelle qui caractérise un fonctionnement de classe s'inspirant du modèle de la communauté d'élaboration de connaissances. Il s'en dégage une répartition plus égalitaire des contributions que dans un modèle pédagogique d'enseignement frontal, où l'essentiel est produit et dispensé par le formateur. On assiste à une décentration de l'attention du formateur 
vers les étudiants, sans pour autant que ce premier perde son importance et sa responsabilité à l'égard du déroulement du cours.

En ce qui a trait spécifiquement aux principes de coélaboration de connaissances, les résultats révèlent que la participation des étudiants s'est articulée en complémentarité. L'analyse de l'usage des marqueurs de relation et du contenu des contributions sur le KF permet de conclure à une dynamique de collaboration plutôt que de compétition ainsi qu'à une amélioration progressive des idées. Par ailleurs, il pourrait être pertinent, dans le cadre d'une prochaine étude, de chercher à comprendre en quoi les idées élaborées ont aiguillé la pratique des étudiants, lorsqu'ils sont retournés enseigner dans leur classe. En outre, à l'exception peutêtre d'un étudiant, les résultats suggèrent que la dynamique mise en place a responsabilisé les étudiants par rapport à leur démarche d'apprentissage, autant de façon individuelle que collective. Plus particulièrement, cette responsabilisation s'est manifestée par la contribution de la grande majorité des participants au développement d'un contenu de groupe. La flexibilité de l'outil et le fait que les étudiants peuvent y contribuer en tout temps ont permis de moduler l'implication de chacun. Enfin, pour ce qui est des principes d'évaluation et de considération de sources fiables, peu de constats peuvent être dégagés puisque les objectifs de l'étude ne les ciblaient pas de façon systématique.

La dynamique de coélaboration de connaissances qui s'est déployée nous apparaît importante pour les raisons suivantes. Tout d'abord, en ayant l'occasion de participer activement au déroulement du cours, cela favorise non seulement un engagement accru des étudiants, mais aussi une appropriation personnelle des contenus. En arrimant cette appropriation à leur pratique actuelle, cela est à même de contribuer au transfert de connaissances. Ensuite, non seulement la participation au sein du cours est-elle active, mais elle convoque les étudiants à effectuer une contribution réelle à un discours de classe conjoint. Il ne s'agit donc plus de répondre aux sollicitations ponctuelles du formateur, mais de prendre part à une démarche d'avancement collective, ce qui favorise une complémentarité de perspectives et de points de vue qui est non seulement bénéfique pour les étudiants, mais aussi pour le responsable du cours. En effet, ce dernier a accès à des éléments contextuels de la pratique de la classe multiâge qui lui permettent d'ajuster son discours pour le rendre davantage viable pour les «formés ». En d'autres mots, cela aiguille et informe son travail de médiation entre les éléments conceptuels et pratiques. Il se produit alors en quelque sorte un recadrage par rapport à la façon habituelle de concevoir la responsabilité de la préparation des professionnels de l'éducation aux défis de demain. Cette responsabilité n'incombe plus essentiellement au formateur universitaire; elle est davantage partagée avec les praticiens qui, par leur participation même au cours, apportent des éléments contextuels susceptibles de compléter avantageusement les principes généraux issus de la recherche.

Un constat additionnel provient de l'analyse des marqueurs de liaison, qui rend compte de la consistance d'un discours caractérisé à la fois par des ajouts et des éléments visant à mettre en perspective des idées. Cela est à même de rassurer certaines personnes pouvant se questionner à propos de la nature des apprentissages effectués dans le contexte d'un tel dispositif pédagogique en enseignement supérieur. Les connaissances relatées dans les résumés rédigés sont certes bien différentes du formalisme qu'on retrouve dans les méthodes d'évaluation classiques. Elles sont plutôt de nature in vivo et rendent compte d'une appropriation personnelle qui est davantage susceptible de traduire un réel changement conceptuel qu'une récitation de faits et procédures bruts mémorisés. C'est d'une connaissance qu'on pourrait qualifier d'incarnée et de située dont il s'agit.

Par ailleurs, la dynamique interactionnelle que renvoie l'analyse du réseau social est complexe et, malgré une plus nette implication globale des étudiants, elle met en lumière certaines disparités entre eux. Tous s'impliquent et participent à l'avancement du discours collectif, mais pas avec la même ardeur. Sur le plan de la gestion de la dynamique de groupe, cela n'est pas sans soulever d'enjeux pour le formateur. Non seulement a-t-il un rôle à jouer pour soutenir un approfondissement des idées, rôle qui passe notamment par la valorisation et l'enrichissement de celles les plus prometteuses formulées par les étudiants. En contrepartie, il doit aussi chercher à ce que tous reçoivent et donnent une attention minimale aux autres, de 
façon à maintenir la collégialité au sein de la communauté. Les sociogrammes ont bien illustré qu'il n'est pas aisé de composer avec un tel enjeu. Et ce ne l'est d'autant pas qu'on cherche à faire fonctionner une communauté en faisant abstraction, au premier plan en tout cas, du pouvoir de contraindre par la sanction évaluative.

Au-delà des résultats qu'elle produit, cette étude nous semble illustrer la pertinence d'outils d'analyse semi-automatisés pour comprendre et modéliser la dynamique qui prévaut au sein d'un cours élaboré à partir de principes d'apprentissage contemporain en enseignement supérieur. Considérant l'ampleur du discours généré par un tel mode d'apprentissage, en tant que formateur, cela soulève un enjeu de temps pour assurer un suivi de qualité. Bien sûr, de tels outils automatiques demeurent imparfaits. Plus spécifiquement en ce qui concerne le contenu des échanges, bien que l'analyse des types de marqueurs de liaison que nous avons proposée ne permette pas de savoir si les idées ajoutées constituent de réels ajouts par rapport aux idées antérieures, et qu'une marge d'erreur assez importante subsiste, elle permet néanmoins d'obtenir un aperçu presque en temps réel du travail effectué par la communauté, ce qui représente une donnée pertinente et intéressante pour alimenter rapidement non seulement le regard du formateur, mais aussi celui des étudiants, et les amener à prendre du recul par rapport à leur démarche d'élaboration d'un discours collectif. Des travaux qui sont en cours au sein de la communauté CSCL à propos de l'analyse sémantique permettent d'espérer obtenir des données qualitatives automatisées de plus en plus riches.

\section{Conclusion}

En guise de conclusion, nous souhaitons nous prémunir d'une critique possible qui pourrait être formulée. Elle concerne le nombre restreint de participants $(\mathrm{N}=8)$ qui ont pris part au cours qui a fait l'objet d'analyse dans le cadre de la rédaction de cet article. D'aucuns pourraient mentionner que la petite taille du groupe a facilité la gestion des interactions entre les individus, en comparaison à ce qui peut être fait dans les groupes de plus grande taille qui caractérisent habituellement les cours en face à face. Certes, il est reconnu que la taille d'un groupe est un facteur pouvant influencer les interactions qui y surviennent. Toutefois, il nous apparaît important de mentionner que le travail en réseau, tout particulièrement celui supporté par un outil de communication asynchrone, offre un potentiel intéressant pour reconsidérer la façon dont les interactions surviennent au sein des grands groupes. Un professeur pourrait par exemple mettre à contribution un tel outil et diviser sa classe en équipes, dont chacune serait responsable d'une thématique en lien avec le sujet général du cours. Nous y voyons une modalité parmi d'autres qui puisse permettre de combiner des interactions en grand groupe ainsi qu'un flux important d'interactions entre étudiants au profit d'une participation plus active, voire créative de leur part.

\section{Références}

Allaire, S., Hamel, C., Gaudreault-Perron, J., \& Laferrière, T. (2012). L'apprentissage collaboratif en réseau au profit de l'intervention en classe multiâge. Revue pour la recherche en éducation.

Allaire, S., \& Lusignan, G. (2011). Enseigner et apprendre en réseau : collaborer entre écoles distantes à l'aide des TIC. Anjou : Éditions CEC.

Allaire, S., Pellerin, G., Beaudoin, M., Couture, C., \& Turcotte, S. (2010). Développement d'un programme de formation interuniversitaire en réseau : pallier une situation découlant des mouvements démographiques au Québec. Journées Communication et Apprentissage Instrumentés en Réseau (JOCAIR 2010), Amiens. [En ligne]. Repéré à : http://affordance.uqac.ca/publications/Texte-JOCAIR2010Final.pdf

Baker-Doyle, K., \& Yoon, S. (2011). In search of practioner-based social capital: A social network analysis tool for understanding and facilitating teacher collaboration in a professional development program. Professional Development in Education, 37, 75-93. 
Béchard, J-P. (2001). L'enseignement supérieur et les innovations pédagogiques : une recension des écrits. Revue des sciences de l'éducation, 27 (2), 257-281.

Bereiter, C., \& Scardamalia, M. (2003). Learning to work creatively with knowledge. Dans E.D. Corte, L. Verschaffel, N. Entwistle, \& J.V. Merriënboer (Éds.), Powerful learning environments: Unravelling basic components and dimensions (p. 73-78). Oxford: Elsevier Science.

Bereiter, C., \& Scardamalia, M. (1989). Intentional learning as a goal of instruction. Dans L. B. Resnick (Ed.), Knowing, learning, and instruction: Essays in honor of Robert Glaser (p. 361-392). Hillsdale, N.J.: Lawrence Erlbaum Associates.

Brady, K., \& Eisler, R. (1999). Sex and gender in the college classroom: A quantitive analysis of faculty-student interactions and perceptions. Journal of Educational Psychology, 91 (1), 127-145.

Bransford, J. D., Brown, A. L., \& Cocking, R. R. (1999). How people learn: Brain, mind, experience, and school. Washington: National Academy Press.

Butler, D. L. (2005). L'autorégulation de l'apprentissage et la collaboration dans le développement professionnel des enseignants. Revue des sciences de l'éducation, 31 (1), 55-78.

Cazden, B. (2001). Classroom discourse: The language of teaching and learning. 2nd Edition. Portsmouth, N.H.: Heinemann.

Cazden, B. (1988). Classroom discourse: The language of teaching and learning. Portsmouth, N.H.: Heinemann Educational Books.

Darling-Hammond, L., \& Bransford, J. (2005). Preparing teachers for a changing world: What teachers should learn and be able to do. San Francisco: Jossey-Bass.

Degenne, A., \& Forsé, M. (1994). Les réseaux sociaux. Une analyse structurale en sociologue. Paris : Armand Colin.

Desgagné, S., Bednarz, N., Couture, C., Poirier, L., \& Lebuis, P. (2001). L'approche collaborative de recherche en éducation : un rapport nouveau à établir entre recherche et formation. Revue des sciences de l'éducation, 27 (1), 33-64.

Desgagné, S., Fortier, A., \& Jobin, I. (2011). Efforts de conciliation de trois «positions de savoir» en formation dans le cadre d'un lien cours/stage en gestion participative de la classe. $7^{e}$ colloque de L'association pour la formation à l'enseignement, Sherbrooke.

Endrizzi, L. (2011). Savoir enseigner dans le supérieur : un enjeu d'excellence pédagogique. Institut français de l'éducation, 64, 1-23.

Garet, M. S., Porter, A. C., Desimone, L., Birman, B. F., \& Yoon, K. S. (2001). What makes professional development effective? Results from a national sample of teachers. American Educational Research Journal, 38 (4), 915-945.

Haythornthwaite, C., \& De Laat, M. (2010). Social Networks and Learning Networks: Using social network perspectives to understand social learning. Proceedings of the 7th International Conference of Networking Learning.

Garet, M. S., Porter, A. C., Desimone, L., Birman, B. F., \& Suk Yoon, K. (2001). What makes professional development effective? Results from a national sample of teachers. American Educational Research Journal, 38 (4), 915-945.

Khine, M., \& Saleh, I. (2010). New science of learning: cognition, computers and collaboration in education. London : Springer.

Kozanitis, A., \& Chouinard, R. (2009). Les facteurs d'influence de la participation verbale en classe des étudiants universitaires : une revue de la littérature. Revue internationale de pédagogie de l'enseignement supérieur, 25 (1), 1-15.

Marcos-García, J. A., Martínez, A., Dimitriadis, Y., \& Anguita, R. (2007). A Role-Based Approach for the Support of Collaborative Learning Activities. e-Services Journal, 6, 4057.

Laferrière, T., \& Allaire, S. (2010). Développement professionnel d'enseignantes et d'enseignants: les passeurs de frontière qui façonnent l'École éloignée en réseau. Éducation - Formation, 293, 1-20. [En ligne.] Disponible : http://ute3.umh.ac.be/revues/include/download.php?idRevue=9\&idRes=66 
Laferrière, T., Hamel, C., Allaire, S., Turcotte, S., Breuleux, A., Beaudoin, J.,... (2011). L'École éloignée en réseau, un modèle. Rapport-synthèse, octobre 2011. CEFRIO.

Laferrière, T. (2005). Les communautés d'apprenants en réseau au bénéfice de l'éducation (Networked learner's communities for the benefit of education). Encounters on Education, 6, 5-21.

Laferrière, T., Lamon, M., \& Breuleux, A. (2006). Blended learning environments. In P. Resta (Ed.), Teacher development in an e-learning age: A policy and planning guide, UNESCO.

Nirula, L., Woodruff, E., Scardamalia, M. \& MacDonald, P. (2003). Handhelds in a grade two classroom: Innovations to support knowledge-building and epistemic agency. In K. Lee \& K. Mitchell (Eds.), Proceedings of the International Conference on Computers in Education 2003 (p. 22-26), Hong Kong.

Palincsar, A. S., Magnussen, S. J., Marano, N., Ford, D., \& Brown, N. (1998). Designing a community of practice: Principles and practices of the GIsML community. Teaching and Teacher Education, 14 (1), 5-19.

Pavoola, S., Lipponen, L. \&, Hakkarainen, K. (2004). Models of innovative knowledge communities and three metaphors of learning. Review of educational research, 74 (4), 557-576.

Penuel, W.R., Riel, M., Krause, A.E., \& Frank, K.A. (2009). Analyzing teacher's professional interactions in a school as social capital: A social network approach. Teachers College Record, 111, 124-163.

Popper, K., \& Eccles, J. (1977). The self and its brain: An argument for interactionism. New York: Springer Verlag.

Ramsden, P. (2003). Learning to teach in higher education. London: Routledge.

Reffay, C. \& Martínez-Monés, A. (sous presse). Basic concepts and techniques in social network analysis. Dans R. Luckin, P. Goodyear, B. Grabowski, S. Puntambeker, J. Underwood, N. Winters (Éds.), Handbook on design educational computing. New York: Taylor \& Francis.

Rennie, L. J. (2001). Teacher collaboration in curriculum change: The implementation of technology education in the primary school. Research in Science Education, 31 (1), 49 69.

Sawyer, K. (Ed.) (2005). The Cambridge handbook of learning sciences. New York: Cambridge University Press.

Scardamalia, M., \& Bereiter, C. (2004). Knowledge Building. In Encyclopedia of Education Second Edition. New York: Macmillan Reference.

Sfard, A. (1998). On two metaphors for learning and on the danger of choosing just one. Educational Researcher, 27 (2), 4-13.

Sinclair, J.M., \& Coulthard, R.M. (1975). Towards an Analysis of Discourse. Oxford: Oxford University Press.

Wasserman, S., \& Faust, K. (1994). Social network analysis: Methods and applications. Cambridge: Cambridge University Press. 